\title{
NUMERICAL APPROXIMATION OF DYNAMIC DEFORMATIONS OF A THERMOVISCOELASTIC ROD AGAINST AN ELASTIC OBSTACLE
}

\author{
Maria I.M. Copetti ${ }^{1}$
}

\begin{abstract}
In this paper we consider a hyperbolic-parabolic problem that models the longitudinal deformations of a thermoviscoelastic rod supported unilaterally by an elastic obstacle. The existence and uniqueness of a strong solution is shown. A finite element approximation is proposed and its convergence is proved. Numerical experiments are reported.
\end{abstract}

Mathematics Subject Classification. 65N30.

Received: May 19, 2003. Revised: March 2 and May 18, 2004.

\section{INTRODUCTION}

We study the following hyperbolic-parabolic system

$$
\begin{gathered}
\theta_{t}-\theta_{x x}=-a u_{x t}, \quad 0<x<1, \quad t>0, \\
b u_{t t}-\sigma_{x}=0, \quad 0<x<1, \quad t>0,
\end{gathered}
$$

where $\sigma=u_{x}+\zeta u_{x t}-a \theta$, with initial conditions

$$
\theta(x, 0)=\theta_{0}(x), \quad u(x, 0)=u_{0}(x), \quad u_{t}(x, 0)=u_{1}(x), \quad 0<x<1,
$$

and boundary conditions

$$
\begin{gathered}
u(0, t)=0, \quad \theta(0, t)=\theta_{A}, \quad-\theta_{x}(1, t)=k \theta(1, t), \quad t>0, \\
\sigma(1, t)=-\frac{1}{\epsilon}[u(1, t)-g]_{+}, \quad t>0,
\end{gathered}
$$

which models the deformations along the $x$-axis of a linear, homogeneous, thermoviscoelastic rod occupying in its reference configuration the interval $I=[0,1]$. The temperature, the axial displacement and the stress of the rod are denoted by $\theta(x, t), u(x, t)$ and $\sigma(x, t)$, respectively. At its left end the rod is clamped and has constant temperature $\theta_{A}$. The right end is free to expand or contract and may be in contact and, possibly, penetrate an elastic obstacle with rigidity $1 / \epsilon>0$ located at distance $g>0$ from the rest position. We assume that there is a heat exchange with the obstacle. Here $a, b \geq 0$ are constants given in terms of physical parameters, $a$ is usually

Keywords and phrases. Thermoviscoelasticity, dynamic contact problem, finite element approximation, numerical simulations.

${ }^{1}$ Laboratório de Análise Numérica e Astrofísica, Departamento de Matemática, Universidade Federal de Santa Maria, 97119-900 Santa Maria, RS, Brazil. e-mail: mimc@lana.ccne.ufsm.br 
small, $\zeta>0$ is the coefficient of viscosity and $k>0$ is the coefficient of heat transfer. When $\epsilon \rightarrow 0$ in (1.5) the resulting boundary condition is the Signorini's condition for contact with a rigid obstacle. In this work, linear viscous effects have been included in the equations of linear thermoelasticity. We refer to Carlson [1], Day [4] and Jiang and Racke [8] for physical background and mathematical modelling.

A related dynamic problem was studied by Elliott and Qi [7]. In that paper, the rod is only thermoelastic $(\zeta=0)$ and a regularization of the problem with elastic obstacle is used to prove existence, without uniqueness, of a weak solution to the limiting case $\epsilon \rightarrow 0$. The existence of weak solutions to the thermoviscoelastic contact problem with a rigid obstacle was shown by Kuttler and Shillor [10] in the case that the heat exchange coefficient is a continuous function or a graph that depends on the distance between the free end and the obstacle.

In order to prove existence and uniqueness of a strong solution to (1.1)-(1.5) it is important the presence of the term $\zeta u_{x t}$ that makes it possible to obtain the necessary a priori estimates to pass to the limit in the associated Fourier-Galerkin formulation. A finite element method is proposed to numerically approximate the present model. Convergence to the solution of the continuous problem is proved and some numerical experiments are presented.

If the acceleration of the rod is small, the term $b u_{t t}$ is usually neglected and the resulting problem becomes quasi-static. This situation was considered by Copetti and French [3] where existence and uniqueness of a strong solution, for both elastic and rigid obstacles, was established and a numerical approximation was proposed and analysed. The quasi-static contact problem for a thermoelastic rod and an elastic obstacle was studied by Copetti [2]. In [9] Kim considers dynamic contact problems with elastic and rigid obstacles for a viscoelastic rod with long memory. Existence of solution to dynamic thermoviscoelastic contact problems in $\mathbb{R}^{n}$ with friction and contact condition for the displacement velocities was proved by Eck [5] and Eck and Jarušek [6]. Numerical approximations by finite element and finite difference methods to the problem that models the deformations of an elastic rod against a rigid obstacle were proposed by Schatzman and Bercovier [11].

Results on dynamic contact problems in thermoelasticity and thermoviscoelasticity modelling the deformations of a rod against a stationary obstacle, elastic or rigid, are scarce and the present work is a contribution to the numerical solution of such problems.

Throughout the paper $C$ denotes positive constants that may depend on data and are not necessarily the same at each occurrence.

\section{EXISTENCE AND UNIQUENESS}

For completeness, we prove in this section that there exists a unique solution to the above problem. We follow similar ideas as in [7] and [3] .

Let us introduce $H_{E}^{1}(I)=\left\{\chi \in H^{1}(I) \mid \chi(0)=0\right\}$ and assume that $b=1$ for simplicity.

Theorem 2.1. Given $\theta_{0} \in H^{2}(I), \theta_{0}(0)=\theta_{A}, \theta_{0}(1)=\theta_{0 x}(1)=0, u_{0} \in H^{2}(I) \cap H_{E}^{1}, u_{0 x}(1)=0, u_{0}(1) \leq g$, and $u_{1} \in H^{2}(I) \cap H_{0}^{1}(I), u_{1 x}(1)=0$, there exists a unique $\{\theta, u\}$ satisfying equations (1.1)-(1.5) with

$$
\begin{gathered}
\theta \in L^{\infty}\left(0, T ; H^{1}(I)\right), \theta_{t}, u_{t t}, \sigma_{x} \in L^{\infty}\left(0, T ; L^{2}(I)\right) \cap L^{2}\left(0, T ; H_{E}^{1}(I)\right), \\
u, u_{t} \in L^{\infty}\left(0, T ; H_{E}^{1}(I)\right), \theta_{x x}, u_{x x}, \sigma \in L^{\infty}\left(0, T ; L^{2}(I)\right) .
\end{gathered}
$$

Proof. Introducing the following change of variables

$$
\tilde{\theta}(x, t)=\theta(x, t)-\theta_{0}(x), \quad \tilde{u}(x, t)=u(x, t)-u_{0}(x)-t u_{1}(x),
$$


it results that $\tilde{\theta}, \tilde{u}$ satisfy, $\forall w, v \in H_{E}^{1}(I)$,

$$
\begin{gathered}
\left(\tilde{\theta}_{t}, w\right)+\left(\tilde{\theta}_{x}+\theta_{0 x}, w_{x}\right)+a\left(\tilde{u}_{x t}+u_{1 x}, w\right)+k \tilde{\theta}(1, t) w(1)=0 \\
\left(\tilde{u}_{t t}, v\right)+\left(\tilde{u}_{x}+\zeta \tilde{u}_{x t}-a \tilde{\theta}-a \theta_{0}+u_{0 x}+(t+\zeta) u_{1 x}, v_{x}\right)+\frac{1}{\epsilon}\left[\tilde{u}(1, t)+u_{0}(1)-g\right]_{+} v(1)=0 .
\end{gathered}
$$

Let $V^{m}=\operatorname{span}\left\{\phi_{i}\right\}_{i=1}^{m}$, where $\left\{\phi_{i}\right\}_{i=1}^{\infty} \subset C^{\infty}(I)$ is an orthogonal basis for $H_{E}^{1}(I)$ and orthonormal for $L^{2}(I)$, and look for

$$
\theta^{m}(x, t)=\sum_{i=1}^{m} c_{i}(t) \phi_{i}(x), \quad u^{m}(x, t)=\sum_{i=1}^{m} d_{i}(t) \phi_{i}(x)
$$

satisfying, $\forall w, v \in V^{m}$,

$$
\begin{gathered}
\left(\theta_{t}^{m}, w\right)+\left(\theta_{x}^{m}+\theta_{0 x}, w_{x}\right)+a\left(u_{x t}^{m}+u_{1 x}, w\right)+k \theta^{m}(1, t) w(1)=0 \\
\left(u_{t t}^{m}, v\right)+\left(u_{x}^{m}+\zeta u_{x t}^{m}-a \theta^{m}-a \theta_{0}+u_{0 x}+(t+\zeta) u_{1 x}, v_{x}\right)+\frac{1}{\epsilon}\left[u^{m}(1, t)+u_{0}(1)-g\right]_{+} v(1)=0
\end{gathered}
$$

with initial conditions $\theta^{m}(x, 0)=u^{m}(x, 0)=u_{t}^{m}(x, 0)=0$. Since the nonlinearity involved is Lipschitz continuous, this initial value problem has a solution on some interval $\left[0, T^{m}\right]$. The a priori estimates established below allow us to conclude that the solution can be extended to the whole interval $[0, T]$ for any given $T$.

Taking $w=\theta^{m}$ and $v=u_{t}^{m}$ we find that

$$
\begin{aligned}
\frac{1}{2} \frac{\mathrm{d}}{\mathrm{d} t}\left\|\theta^{m}\right\|^{2}+ & \left\|\theta_{x}^{m}\right\|^{2}+k\left(\theta^{m}(1, t)\right)^{2}+\frac{1}{2} \frac{\mathrm{d}}{\mathrm{d} t}\left\|u_{t}^{m}\right\|^{2}+\frac{1}{2} \frac{\mathrm{d}}{\mathrm{d} t}\left\|u_{x}^{m}\right\|^{2}+\zeta\left\|u_{x t}^{m}\right\|^{2}+\frac{1}{2 \epsilon} \frac{\mathrm{d}}{\mathrm{d} t}\left[u^{m}(1, t)+u_{0}(1)-g\right]_{+}^{2} \\
= & -\left(\theta_{0 x}, \theta_{x}^{m}\right)-a\left(u_{1 x}, \theta^{m}\right)+\left(a \theta_{0}-u_{0 x}-(t+\zeta) u_{1 x}, u_{x t}^{m}\right) \leq C+\frac{1}{2}\left(\left\|\theta_{x}^{m}\right\|^{2}+\left\|\theta^{m}\right\|^{2}+\zeta\left\|u_{x t}^{m}\right\|^{2}\right)
\end{aligned}
$$

Thus, Gronwall's inequality implies that

$$
\begin{gathered}
\left\|\theta^{m}(\cdot, T)\right\|^{2}+\int_{0}^{T}\left\|\theta_{x}^{m}\right\|^{2} \mathrm{~d} t+k \int_{0}^{T}\left(\theta^{m}(1, t)\right)^{2} \mathrm{~d} t \\
+\left\|u_{t}^{m}(\cdot, T)\right\|^{2}+\left\|u_{x}^{m}(\cdot, T)\right\|^{2}+\zeta \int_{0}^{T}\left\|u_{x t}^{m}\right\|^{2} \mathrm{~d} t \\
+\frac{1}{\epsilon}\left[u^{m}(1, T)+u_{0}(1)-g\right]_{+}^{2} \leq C .
\end{gathered}
$$

Substituting $\theta_{t}^{m}$ for $w$ gives

$$
\begin{aligned}
\left\|\theta_{t}^{m}\right\|^{2}+\frac{1}{2} \frac{\mathrm{d}}{\mathrm{d} t}\left\|\theta_{x}^{m}\right\|^{2}+\frac{k}{2} \frac{\mathrm{d}}{\mathrm{d} t}\left(\theta^{m}(1, t)\right)^{2} & =-\left(\theta_{0 x}, \theta_{x t}^{m}\right)-a\left(u_{x t}^{m}+u_{1 x}, \theta_{t}^{m}\right) \\
& \leq-\frac{\mathrm{d}}{\mathrm{d} t}\left(\theta_{0 x}, \theta_{x}^{m}\right)+a^{2}\left(\left\|u_{x t}^{m}\right\|^{2}+\left\|u_{1 x}\right\|^{2}\right)+\frac{1}{2}\left\|\theta_{t}^{m}\right\|^{2} .
\end{aligned}
$$

By virtue of the previous estimate we deduce that

$$
\int_{0}^{T}\left\|\theta_{t}^{m}\right\|^{2} \mathrm{~d} t+\left\|\theta_{x}^{m}(\cdot, T)\right\|^{2}+k\left(\theta^{m}(1, T)\right)^{2} \leq C .
$$


Differentiating equations (2.1)-(2.2) with respect to $t$ and setting $w=\theta_{t}^{m}, v=u_{t t}^{m}$ it results

$$
\begin{gathered}
\frac{1}{2} \frac{\mathrm{d}}{\mathrm{d} t}\left\|\theta_{t}^{m}\right\|^{2}+\left\|\theta_{x t}^{m}\right\|^{2}+k\left(\theta_{t}^{m}(1, t)\right)^{2}+a\left(u_{x t t}^{m}, \theta_{t}^{m}\right)=0, \\
\frac{1}{2} \frac{\mathrm{d}}{\mathrm{d} t}\left(\left\|u_{t t}^{m}\right\|^{2}+\left\|u_{x t}^{m}\right\|^{2}\right)+\zeta\left\|u_{x t t}^{m}\right\|^{2}-a\left(\theta_{t}^{m}, u_{x t t}^{m}\right)+\frac{1}{\epsilon} \frac{\mathrm{d}}{\mathrm{d} t}\left[u^{m}(1, t)+u_{0}(1)-g\right]_{+} u_{t t}^{m}(1, t) \\
=-\left(u_{1 x}, u_{x t t}^{m}\right) \leq \frac{1}{2 \zeta}\left\|u_{1 x}\right\|^{2}+\frac{\zeta}{2}\left\|u_{x t t}^{m}\right\|^{2} .
\end{gathered}
$$

Hence, for $\delta>0$,

$\frac{1}{2} \frac{\mathrm{d}}{\mathrm{d} t}\left\|\theta_{t}^{m}\right\|^{2}+\left\|\theta_{x t}^{m}\right\|^{2}+k\left(\theta_{t}^{m}(1, t)\right)^{2}+\frac{1}{2} \frac{\mathrm{d}}{\mathrm{d} t}\left(\left\|u_{t t}^{m}\right\|^{2}+\left\|u_{x t}^{m}\right\|^{2}\right)+\frac{\zeta}{2}\left\|u_{x t t}^{m}\right\|^{2} \leq C+\frac{1}{2 \epsilon^{2} \delta}\left(u_{t}^{m}(1, t)\right)^{2}+\frac{\delta}{2}\left(u_{t t}^{m}(1, t)\right)^{2}$.

Observing that

$$
u_{t}^{m}(1, t)=\int_{0}^{1} u_{x t}^{m} \mathrm{~d} x, \quad u_{t t}^{m}(1, t)=\int_{0}^{1} u_{x t t}^{m} \mathrm{~d} x,
$$

and taking $\delta=\zeta / 2$ we obtain, recalling that $\int_{0}^{T}\left\|u_{x t}^{m}\right\|^{2} \mathrm{~d} t \leq C$,

$$
\begin{aligned}
& \frac{1}{2}\left\|\theta_{t}^{m}(\cdot, T)\right\|^{2}+\int_{0}^{T}\left\|\theta_{x t}^{m}\right\|^{2} \mathrm{~d} t+k \int_{0}^{T}\left(\theta_{t}^{m}(1, t)\right)^{2} \mathrm{~d} t+\frac{1}{2}\left\|u_{t t}^{m}(\cdot, T)\right\|^{2} \\
& \quad+\frac{1}{2}\left\|u_{x t}^{m}(\cdot, T)\right\|^{2}+\frac{\zeta}{4} \int_{0}^{T}\left\|u_{x t t}^{m}\right\|^{2} \mathrm{~d} t \leq C+\frac{1}{2}\left(\left\|\theta_{t}^{m}(\cdot, 0)\right\|^{2}+\left\|u_{t t}^{m}(\cdot, 0)\right\|^{2}\right) .
\end{aligned}
$$

We need to estimate $\left\|\theta_{t}^{m}(\cdot, 0)\right\|$ and $\left\|u_{t t}^{m}(\cdot, 0)\right\|$. Letting $t=0$ in equations (2.1) and (2.2) we get $\forall w, v \in V^{m}$,

$$
\begin{gathered}
\left(\theta_{t}^{m}(\cdot, 0), w\right)=-\left(\theta_{0 x}, w_{x}\right)-a\left(u_{1 x}, w\right)=\left(\theta_{0 x x}, w\right)-a\left(u_{1 x}, w\right), \\
\left(u_{t t}^{m}(\cdot, 0), v\right)=\left(a \theta_{0}-u_{0 x}-\zeta u_{1 x}, v_{x}\right)=-\left(a \theta_{0 x}-u_{0 x x}-\zeta u_{1 x x}, v\right),
\end{gathered}
$$

and it follows that $\theta_{t}^{m}(\cdot, 0)$ and $u_{t t}^{m}(\cdot, 0)$ are bounded in $L^{2}(I)$. Using the above estimates, we can select subsequences, denoted by $\left\{\theta^{m}\right\},\left\{u^{m}\right\}$, such that

$$
\begin{gathered}
\theta^{m} \rightarrow \tilde{\theta}, u^{m} \rightarrow \tilde{u}, u_{t}^{m} \rightarrow \tilde{u}_{t} \text { weakly } * \text { in } L^{\infty}\left(0, T ; H_{E}^{1}(I)\right), \\
\theta_{t}^{m} \rightarrow \tilde{\theta}_{t} \text { weakly } * \text { in } L^{\infty}\left(0, T ; L^{2}(I)\right) \text { and weakly in } L^{2}\left(0, T ; H_{E}^{1}(I)\right), \\
u_{t t}^{m} \rightarrow \tilde{u}_{t t} \text { weakly * in } L^{\infty}\left(0, T ; L^{2}(I)\right) \text { and weakly in } L^{2}\left(0, T ; H_{E}^{1}(I)\right), \\
\theta^{m}(1, \cdot) \rightarrow \tilde{\theta}(1, \cdot) \text { weakly } * \text { in } L^{\infty}(0, T) .
\end{gathered}
$$

We infer also that $u^{m}(1, \cdot) \rightarrow \tilde{u}(1, \cdot)$ in $H^{1}(0, T)$ weakly, and the fact that the injection of $H^{1}(0, T)$ into $L^{2}(0, T)$ is compact implies that $u^{m}(1, \cdot) \rightarrow \tilde{u}(1, \cdot)$ in $L^{2}(0, T)$ strongly. Noting that

$$
\left\|\left[u^{m}(1, \cdot)+u_{0}(1)-g\right]_{+}-\left[\tilde{u}(1, \cdot)+u_{0}(1)-g\right]_{+}\right\|_{L^{2}(0, T)} \leq\left\|u^{m}(1, \cdot)-\tilde{u}(1, \cdot)\right\|_{L^{2}(0, T)}
$$


we deduce that

$$
\left[u^{m}(1, \cdot)+u_{0}(1)-g\right]_{+} \rightarrow\left[\tilde{u}(1, \cdot)+u_{0}(1)-g\right]_{+} \text {in } L^{2}(0, T) \text { strongly. }
$$

We can then pass to the limit and reverse the change of variables to find that

$$
\begin{gathered}
\left(\theta_{t}, w\right)+\left(\theta_{x}, w_{x}\right)+a\left(u_{x t}, w\right)+k \theta(1, t) w(1)=0, \\
\left(u_{t t}, v\right)+\left(u_{x}+\zeta u_{x t}-a \theta, v_{x}\right)+\frac{1}{\epsilon}[u(1, t)-g]_{+} v(1)=0
\end{gathered}
$$

hold for every $w, v \in H_{E}^{1}(I)$. By the standard argument, the existence result follows. The argument used by Copetti and French in [3] yields the $H^{2}$ regularity for $u(\cdot, t)$ : from the defining equations,

$$
u_{x}+\zeta u_{x t}=a \theta+\sigma
$$

and therefore,

Integrating in time we obtain

$$
\frac{\mathrm{d}}{\mathrm{d} t}\left(\mathrm{e}^{t / \zeta} u_{x}(\cdot, t)\right)=\frac{1}{\zeta}(a \theta(\cdot, t)+\sigma(\cdot, t)) \mathrm{e}^{t / \zeta}
$$

$$
u_{x}(\cdot, t)=\mathrm{e}^{-t / \zeta}\left(u_{x}(\cdot, 0)+\frac{1}{\zeta} \int_{0}^{t}(a \theta(\cdot, s)+\sigma(\cdot, s)) \mathrm{e}^{s / \zeta} \mathrm{d} s\right)
$$

and differentiating with respect to $x$ we find that $u_{x x}(\cdot, t) \in L^{2}(I)$.

Let us suppose that $\left\{\theta^{1}, u^{1}\right\}$ and $\left\{\theta^{2}, u^{2}\right\}$ are two solutions. Setting $\theta=\theta^{1}-\theta^{2}, u=u^{1}-u^{2}$, and choosing $w=\theta$ and $v=u_{t}$ in the weak form above, we have

$\frac{1}{2} \frac{\mathrm{d}}{\mathrm{d} t}\left(\|\theta\|^{2}+\left\|u_{t}\right\|^{2}+\left\|u_{x}\right\|^{2}\right)+\left\|\theta_{x}\right\|^{2}+k(\theta(1, t))^{2}+\zeta\left\|u_{x t}\right\|^{2}+\frac{1}{\epsilon}\left(\left[u^{1}(1, t)-g\right]_{+}-\left[u^{2}(1, t)-g\right]_{+}\right) u_{t}(1, t)=0$.

Observing that

$$
\left|\frac{1}{\epsilon}\left(\left[u^{1}(1, t)-g\right]_{+}-\left[u^{2}(1, t)-g\right]_{+}\right) u_{t}(1, t)\right| \leq C\left\|u_{x}\right\|^{2}+\frac{\zeta}{2}\left\|u_{x t}\right\|^{2}
$$

the uniqueness of the solution follows from Gronwall's inequality.

Remark 2.2. Let us observe that a generalized energy associated to the model considered here decays as time tends to infinity. Substituting $\hat{\theta}(x, t)=\theta(x, t)+\theta_{A} r(x)$, with $r(x)=c x-1, c=k /(k+1)$, in $(1.1)-(1.2)$ we find that $\{\hat{\theta}, u\}$ satisfies, $\forall w, v \in H_{E}^{1}(I)$,

$$
\begin{gathered}
\left(\hat{\theta}_{t}, w\right)+\left(\hat{\theta}_{x}, w_{x}\right)+a\left(u_{x t}, w\right)+k \hat{\theta}(1, t) w(1)=0 \\
\left(u_{t t}, v\right)+\left(u_{x}+\zeta u_{x t}-a \hat{\theta}+a \theta_{A} r, v_{x}\right)+\frac{1}{\epsilon}[u(1, t)-g]_{+} v(1)=0 .
\end{gathered}
$$

Choosing $w=\hat{\theta}$ and $v=u_{t}$ it results that $\hat{E}(t)=\frac{1}{2}\left(\|\hat{\theta}\|^{2}+\left\|u_{t}\right\|^{2}+\left\|u_{x}\right\|^{2}+\frac{1}{\epsilon}[u(1, t)-g]_{+}^{2}\right)+a \theta_{A}\left(r, u_{x}\right)$ satisfies

$$
\frac{\mathrm{d}}{\mathrm{d} t} \hat{E}(t) \leq 0
$$

which implies that

$$
\frac{\mathrm{d}}{\mathrm{d} t}\left(\frac{1}{2}\left(\|\theta\|^{2}+\left\|u_{t}\right\|^{2}+\left\|u_{x}\right\|^{2}+\frac{1}{\epsilon}[u(1, t)-g]_{+}^{2}\right)+\theta_{A}(r, \theta)+a \theta_{A}\left(r, u_{x}\right)\right) \leq 0 .
$$




\section{NumERICAL APPROXIMATION}

In this section we extend the numerical scheme of Copetti and French [3] to the present situation.

We denote by $S_{E}^{h} \subset H_{E}^{1}(I)$ the space of continuous piecewise linear functions defined on a equidistant partition $0=x_{0}<x_{1}<\ldots<x_{s}=1$ of $I$ into subintervals of length $h=1 / s$ and, given $v \in H_{E}^{1}(I)$, we indicate by $P_{E}^{h} v$ the $H_{E}^{1}$ projection onto $S_{E}^{h}$ defined by $\left(\left(P_{E}^{h} v-v\right)_{x}, \eta_{x}\right)=0 \forall \eta \in S_{E}^{h}$, which satisfies

$$
P_{E}^{h} v \rightarrow v \text { in } H_{E}^{1}(I) \text { strongly as } h \rightarrow 0, \quad\left\|\left(P_{E}^{h} v\right)_{x}\right\| \leq\left\|v_{x}\right\|, \quad P_{E}^{h} v\left(x_{i}\right)=v\left(x_{i}\right) .
$$

The Galerkin approximation to (2.3)-(2.4) is to find $\Theta^{n}, U^{n} \in S_{E}^{h}$, such that, $\forall w, v \in S_{E}^{h}$,

$$
\begin{gathered}
\frac{1}{\Delta t}\left(\Theta^{n}-\Theta^{n-1}, w\right)+\left(\Theta_{x}^{n}, w_{x}\right)+\frac{a}{\Delta t}\left(U_{x}^{n}-U_{x}^{n-1}, w\right)+k \Theta^{n}(1) w(1)=0, \quad n=1, \ldots, N, \\
\frac{1}{(\Delta t)^{2}}\left(U^{n}-2 U^{n-1}+U^{n-2}, v\right)+\left(U_{x}^{n}-a \Theta^{n}+a \theta_{A} r, v_{x}\right)+\frac{\zeta}{\Delta t}\left(U_{x}^{n}-U_{x}^{n-1}, v_{x}\right) \\
+\frac{1}{\epsilon}\left[U^{n}(1)-g\right]_{+} v(1)=0, \quad n=2, \ldots, N,
\end{gathered}
$$

where $\Theta^{0}=P_{E}^{h}\left(\theta_{0}+\theta_{A} r\right), U^{0}=P_{E}^{h} u_{0}, U^{1}=U^{0}+\Delta t P_{E}^{h} u_{1}$ and $\Delta t=T / N$.

To implement (3.1)-(3.2) for $n=2, \ldots, N$, we need to solve two coupled systems of equations at each time step and the following iterative procedure was used:

$$
\begin{gathered}
\frac{1}{\Delta t}\left(\Theta^{n, l}-\Theta^{n-1}, w\right)+\left(\Theta_{x}^{n, l}, w_{x}\right)+\frac{a}{\Delta t}\left(U_{x}^{n, l-1}-U_{x}^{n-1}, w\right)+k \Theta^{n, l}(1) w(1)=0, \\
\frac{1}{(\Delta t)^{2}}\left(U^{n, l}-2 U^{n-1}+U^{n-2}, v\right)+\left(U_{x}^{n, l}-a \Theta^{n, l}+a \theta_{A} r, v_{x}\right)+\frac{\zeta}{\Delta t}\left(U_{x}^{n, l}-U_{x}^{n-1}, v_{x}\right) \\
+\frac{1}{\epsilon}\left[U^{n, l-1}(1)-g\right]_{+} v(1)=0,
\end{gathered}
$$

where $\Theta^{n, 0}=\Theta^{n-1}$ and $U^{n, 0}=U^{n-1}$.

Defining

$$
\Theta^{n}=\sum_{i=1}^{s} c_{i}^{n} \eta_{i}, \quad U^{n}=\sum_{i=1}^{s} d_{i}^{n} \eta_{i},
$$

with $\left\{\eta_{i}\right\}_{i=1}^{s}$ the usual basis for $S_{E}^{h}$, we find that $\underline{c}^{n, l}$ and $\underline{d}^{n, l}$ solve the algebraic systems

$$
\begin{aligned}
(M+\Delta t K+\Delta t k B) \underline{c}^{n, l}= & M \underline{\underline{c}}^{n-1}+a C\left(\underline{d}^{n-1}-\underline{d}^{n, l-1}\right), \\
\left(M+\left((\Delta t)^{2}+\Delta t \zeta\right) K\right) \underline{d}^{n, l}= & (2 M+\Delta t \zeta K) \underline{d}^{n-1}-M \underline{d}^{n-2}-(\Delta t)^{2} a \theta_{A} \underline{z} \\
& +(\Delta t)^{2} a C^{T} \underline{c}^{n, l}-(\Delta t)^{2}\left[d_{s}^{n, l-1}-g\right]_{+} \underline{e} / \epsilon,
\end{aligned}
$$

where

$$
M_{i j}=\left(\eta_{i}, \eta_{j}\right), K_{i j}=\left(\eta_{i x}, \eta_{j x}\right), B_{i j}=\eta_{i}(1) \eta_{j}(1), C_{i j}=\left(\eta_{i}, \eta_{j x}\right),\{\underline{z}\}_{i}=\left(r, \eta_{i x}\right) .
$$

Convergence as $l \rightarrow \infty$ of the sequences generated by (3.3)-(3.4) to the unique solution of (3.1)-(3.2), for $a<1$ and $\Delta t<\epsilon \zeta$, follows as in the work of Copetti and French [3]. 


\section{Stability}

It is our aim in this section to derive general a priori estimates for our discrete scheme.

Theorem 4.1. The sequences $\left\{\Theta^{n}, U^{n}\right\}$ generated by (3.1)-(3.2) satisfy the stability estimate

$$
\begin{aligned}
\sum_{i=2}^{n}\left\|\Theta^{i}-\Theta^{i-1}\right\|^{2}+\left\|\Theta^{n}\right\|^{2}+\Delta t \sum_{i=2}^{n}\left\|\Theta_{x}^{i}\right\|^{2} & +k \Delta t \sum_{i=2}^{n}\left(\Theta^{i}(1)\right)^{2}+\sum_{i=2}^{n}\left\|\delta_{u}^{i}-\delta_{u}^{i-1}\right\|^{2}+\left\|\delta_{u}^{n}\right\|^{2} \\
& +\Delta t \zeta \sum_{i=2}^{n}\left\|\delta_{u x}^{i}\right\|^{2}+\sum_{i=2}^{n}\left\|U_{x}^{i}-U_{x}^{i-1}\right\|^{2}+\left\|U_{x}^{n}\right\|^{2}+\frac{1}{\epsilon}\left[U^{n}(1)-g\right]_{+}^{2} \leq C
\end{aligned}
$$

where $\delta_{u}^{n}=\left(U^{n}-U^{n-1}\right) / \Delta t$ for $n \geq 1$ and $C>0$ is a constant independent of $h$ and $\Delta t$.

Proof. Taking $w=\Delta t \Theta^{n}$ in (3.1), $v=\Delta t \delta_{u}^{n}$ in (3.2) and adding the resulting equations yields

$$
\begin{array}{r}
\frac{1}{2}\left(\left\|\Theta^{n}-\Theta^{n-1}\right\|^{2}+\left\|\Theta^{n}\right\|^{2}-\left\|\Theta^{n-1}\right\|^{2}\right)+\Delta t\left\|\Theta_{x}^{n}\right\|^{2}+k \Delta t\left(\Theta^{n}(1)\right)^{2}+\frac{1}{2}\left(\left\|\delta_{u}^{n}-\delta_{u}^{n-1}\right\|^{2}+\left\|\delta_{u}^{n}\right\|^{2}-\left\|\delta_{u}^{n-1}\right\|^{2}\right) \\
+\frac{1}{2}\left(\left\|U_{x}^{n}-U_{x}^{n-1}\right\|^{2}+\left\|U_{x}^{n}\right\|^{2}-\left\|U_{x}^{n-1}\right\|^{2}\right)+\Delta t \zeta\left\|\delta_{u x}^{n}\right\|^{2}+\frac{\Delta t}{\epsilon}\left[U^{n}(1)-g\right]_{+} \delta_{u}^{n}(1) \\
=-\Delta t a \theta_{A}\left(r, \delta_{u x}^{n}\right) \leq C \Delta t+\frac{\Delta t}{2} \zeta\left\|\delta_{u x}^{n}\right\|^{2} .
\end{array}
$$

Noting that

$$
\begin{array}{r}
\Delta t\left[U^{n}(1)-g\right]_{+} \delta_{u}^{n}(1)=\left[U^{n}(1)-g\right]_{+}\left(U^{n}(1)-g+g-U^{n-1}(1)\right)=\left[U^{n}(1)-g\right]_{+}^{2}-\left[U^{n}(1)-g\right]_{+}\left(U^{n-1}(1)-g\right) \\
\geq\left[U^{n}(1)-g\right]_{+}^{2}-\left[U^{n}(1)-g\right]_{+}\left[U^{n-1}(1)-g\right]_{+} \geq \frac{1}{2}\left(\left[U^{n}(1)-g\right]_{+}^{2}-\left[U^{n-1}(1)-g\right]_{+}^{2}\right)
\end{array}
$$

and summing over $n$, we find

$$
\begin{array}{r}
\frac{1}{2}\left(\sum_{i=2}^{n}\left\|\Theta^{i}-\Theta^{i-1}\right\|^{2}+\left\|\Theta^{n}\right\|^{2}\right)+\Delta t \sum_{i=2}^{n}\left(\left\|\Theta_{x}^{i}\right\|^{2}+k\left(\Theta^{i}(1)\right)^{2}\right)+\frac{1}{2}\left(\sum_{i=2}^{n}\left\|\delta_{u}^{i}-\delta_{u}^{i-1}\right\|^{2}+\left\|\delta_{u}^{n}\right\|^{2}\right) \\
+\frac{1}{2}\left(\sum_{i=2}^{n}\left\|U_{x}^{i}-U_{x}^{i-1}\right\|^{2}+\left\|U_{x}^{n}\right\|^{2}\right)+\frac{\Delta t \zeta}{2} \sum_{i=2}^{n}\left\|\delta_{u x}^{i}\right\|^{2}+\frac{1}{2 \epsilon}\left[U^{n}(1)-g\right]_{+}^{2} \\
\leq C+\frac{1}{2}\left(\left\|\Theta^{1}\right\|^{2}+\left\|\delta_{u}^{1}\right\|^{2}+\left\|U_{x}^{1}\right\|^{2}\right)+\frac{1}{2 \epsilon}\left[U^{1}(1)-g\right]_{+}^{2} .
\end{array}
$$

It follows from the definition of $U^{0}, U^{1}$ and the properties of $P_{E}^{h}$ that the last three terms on the right hand side of the above inequality are bounded. It remains to estimate $\left\|\Theta^{1}\right\|$. We have

$$
\begin{aligned}
\frac{1}{2}\left(\left\|\Theta^{1}-\Theta^{0}\right\|^{2}+\left\|\Theta^{1}\right\|^{2}-\left\|\Theta^{0}\right\|^{2}\right)+\Delta t\left\|\Theta_{x}^{1}\right\|^{2}+k \Delta t\left(\Theta^{1}(1)\right)^{2} & =-a\left(U_{x}^{1}-U_{x}^{0}, \Theta^{1}\right) \\
& \leq a^{2}\left\|U_{x}^{1}-U_{x}^{0}\right\|^{2}+\frac{1}{4}\left\|\Theta^{1}\right\|^{2}
\end{aligned}
$$

and hence $\left\|\Theta^{1}\right\| \leq C$. 
Corollary 4.2. We have that

$$
\Delta t \sum_{i=1}^{n}\left\|\frac{\Theta^{i}-\Theta^{i-1}}{\Delta t}\right\|^{2}+\sum_{i=1}^{n}\left\|\Theta_{x}^{i}-\Theta_{x}^{i-1}\right\|^{2}+\left\|\Theta_{x}^{n}\right\|^{2}+k \sum_{i=1}^{n}\left(\Theta^{i}(1)-\Theta^{i-1}(1)\right)^{2}+k\left(\Theta^{n}(1)\right)^{2} \leq C
$$

Proof. Setting $w=\Theta^{n}-\Theta^{n-1}$ in (3.1) gives

$$
\begin{array}{r}
\frac{1}{\Delta t}\left\|\Theta^{n}-\Theta^{n-1}\right\|^{2}+\frac{1}{2}\left(\left\|\Theta_{x}^{n}-\Theta_{x}^{n-1}\right\|^{2}+\right. \\
\left.\left\|\Theta_{x}^{n}\right\|^{2}-\left\|\Theta_{x}^{n-1}\right\|^{2}\right)+\frac{k}{2}\left(\left(\Theta^{n}(1)-\Theta^{n-1}(1)\right)^{2}+\left(\Theta^{n}(1)\right)^{2}-\left(\Theta^{n-1}(1)\right)^{2}\right) \\
=-\frac{a}{\Delta t}\left(U_{x}^{n}-U_{x}^{n-1}, \Theta^{n}-\Theta^{n-1}\right) \leq \frac{a^{2} \Delta t}{2}\left\|\delta_{u x}^{n}\right\|^{2}+\frac{1}{2 \Delta t}\left\|\Theta^{n}-\Theta^{n-1}\right\|^{2}
\end{array}
$$

and the desired estimate is now a consequence of the previous Theorem after summation over $n$. We also used that $\Delta t\left\|\delta_{u x}^{1}\right\|^{2} \leq T\left\|u_{1 x}\right\|^{2}$.

Theorem 4.3. There exists a positive constant $C$, independent of $h$ and $\Delta t$, such that

$$
\begin{aligned}
\sum_{i=2}^{n}\left\|\delta_{\theta}^{i+1}-\delta_{\theta}^{i}\right\|^{2}+\left\|\delta_{\theta}^{n+1}\right\|^{2}+\Delta t \sum_{i=2}^{n}\left\|\delta_{\theta x}^{i+1}\right\|^{2}+k \Delta t \sum_{i=2}^{n}\left(\delta_{\theta}^{i+1}(1)\right)^{2}+\sum_{i=2}^{n}\left\|\gamma^{i+1}-\gamma^{i}\right\|^{2}+\left\|\gamma^{n+1}\right\|^{2} & \\
& +\sum_{i=2}^{n}\left\|\delta_{u x}^{i+1}-\delta_{u x}^{i}\right\|^{2}+\left\|\delta_{u x}^{n+1}\right\|^{2}+\zeta \Delta t \sum_{i=2}^{n}\left\|\gamma_{x}^{i+1}\right\|^{2} \leq C
\end{aligned}
$$

where $\gamma^{n}=\left(\delta_{u}^{n}-\delta_{u}^{n-1}\right) / \Delta t$ for $n \geq 2$.

Proof. Writing equations (3.1) and (3.2) for $n+1$ it follows that, for all $w, v \in S_{E}^{h}$,

$$
\begin{gathered}
\left(\delta_{\theta}^{n+1}-\delta_{\theta}^{n}, w\right)+\Delta t\left(\delta_{\theta x}^{n+1}, w_{x}\right)+a \Delta t\left(\gamma_{x}^{n+1}, w\right)+k \Delta t \delta_{\theta}^{n+1}(1) w(1)=0 \\
\left(\gamma^{n+1}-\gamma^{n}, v\right)+\Delta t\left(\delta_{u x}^{n+1}-a \delta_{\theta}^{n+1}+\zeta \gamma_{x}^{n+1}, v_{x}\right)+\frac{1}{\epsilon}\left(\left[U^{n+1}(1)-g\right]_{+}-\left[U^{n}(1)-g\right]_{+}\right) v(1)=0 .
\end{gathered}
$$

Choosing $w=\delta_{\theta}^{n+1}, v=\gamma^{n+1}$ and adding the resulting equations yields

$$
\begin{aligned}
\frac{1}{2}\left(\left\|\delta_{\theta}^{n+1}-\delta_{\theta}^{n}\right\|^{2}+\left\|\delta_{\theta}^{n+1}\right\|^{2}-\|\right. & \left.\delta_{\theta}^{n} \|^{2}\right)+\Delta t\left\|\delta_{\theta x}^{n+1}\right\|^{2}+k \Delta t\left(\delta_{\theta}^{n+1}(1)\right)^{2}+\frac{1}{2}\left(\left\|\gamma^{n+1}-\gamma^{n}\right\|^{2}+\left\|\gamma^{n+1}\right\|^{2}-\left\|\gamma^{n}\right\|^{2}\right) \\
+\Delta t\left(\delta_{u x}^{n+1}, \gamma_{x}^{n+1}\right)+\zeta \Delta t\left\|\gamma_{x}^{n+1}\right\|^{2} & =-\frac{1}{\epsilon}\left(\left[U^{n+1}(1)-g\right]_{+}-\left[U^{n}(1)-g\right]_{+}\right) \gamma^{n+1}(1)
\end{aligned}
$$

Since

$$
\begin{aligned}
\frac{1}{\epsilon}\left|\left(\left[U^{n+1}(1)-g\right]_{+}-\left[U^{n}(1)-g\right]_{+}\right)\right|\left|\gamma^{n+1}(1)\right| & \leq \frac{1}{\epsilon}\left|U^{n+1}(1)-U^{n}(1) \| \gamma^{n+1}(1)\right| \\
& \leq \frac{\Delta t}{2 \epsilon^{2} \zeta}\left\|\delta_{u x}^{n+1}\right\|^{2}+\frac{\zeta \Delta t}{2}\left\|\gamma_{x}^{n+1}\right\|^{2}
\end{aligned}
$$


and $\Delta t\left(\delta_{u x}^{n+1}, \gamma_{x}^{n+1}\right)=\left(\delta_{u x}^{n+1}, \delta_{u x}^{n+1}-\delta_{u x}^{n}\right)$ we find, after summation over $n$ and using Theorem 4.1,

$$
\begin{aligned}
\frac{1}{2} \sum_{i=2}^{n}\left\|\delta_{\theta}^{i+1}-\delta_{\theta}^{i}\right\|^{2}+ & \frac{1}{2}\left\|\delta_{\theta}^{n+1}\right\|^{2}+\Delta t \sum_{i=2}^{n}\left\|\delta_{\theta x}^{i+1}\right\|^{2}+k \Delta t \sum_{i=2}^{n}\left(\delta_{\theta}^{i+1}(1)\right)^{2}+\frac{1}{2} \sum_{i=2}^{n}\left\|\gamma^{i+1}-\gamma^{i}\right\|^{2}+\frac{1}{2}\left\|\gamma^{n+1}\right\|^{2} \\
& +\frac{1}{2} \sum_{i=2}^{n}\left\|\delta_{u x}^{i+1}-\delta_{u x}^{i}\right\|^{2}+\frac{1}{2}\left\|\delta_{u x}^{n+1}\right\|^{2}+\frac{\zeta}{2} \Delta t \sum_{i=2}^{n}\left\|\gamma_{x}^{i+1}\right\|^{2} \leq C+\frac{1}{2}\left(\left\|\delta_{\theta}^{2}\right\|^{2}+\left\|\gamma^{2}\right\|^{2}+\left\|\delta_{u x}^{2}\right\|^{2}\right) .
\end{aligned}
$$

Let us estimate the last three terms on the right hand side. We have, from equations (3.1) and (3.2),

$$
\begin{gathered}
\frac{1}{2}\left(\left\|\delta_{\theta}^{2}-\delta_{\theta}^{1}\right\|^{2}+\left\|\delta_{\theta}^{2}\right\|^{2}-\left\|\delta_{\theta}^{1}\right\|^{2}\right)+\Delta t\left\|\delta_{\theta x}^{2}\right\|^{2}+k \Delta t\left(\delta_{\theta}^{2}(1)\right)^{2}+a \Delta t\left(\gamma_{x}^{2}, \delta_{\theta}^{2}\right)=0 \\
\left\|\gamma^{2}\right\|^{2}+\left(U_{x}^{2}-a \Theta^{2}+a \theta_{A} r+\zeta \delta_{u x}^{2}, \gamma_{x}^{2}\right)+\frac{1}{\epsilon}\left[U^{2}(1)-g\right]_{+} \gamma^{2}(1)=0
\end{gathered}
$$

Substituting $U^{2}=U^{1}+\Delta t \delta_{u}^{2}, \Theta^{2}=\Theta^{1}+\Delta t \delta_{\theta}^{2}$ and $\delta_{u}^{2}=\delta_{u}^{1}+\Delta t \gamma^{2}$ gives

$$
\begin{aligned}
\frac{1}{2}\left(\left\|\delta_{\theta}^{2}-\delta_{\theta}^{1}\right\|^{2}+\right. & \left.\left\|\delta_{\theta}^{2}\right\|^{2}-\left\|\delta_{\theta}^{1}\right\|^{2}\right)+\Delta t\left\|\delta_{\theta x}^{2}\right\|^{2}+k \Delta t\left(\delta_{\theta}^{2}(1)\right)^{2}+\left\|\gamma^{2}\right\|^{2}+\zeta \Delta t\left\|\gamma_{x}^{2}\right\|^{2} \\
& +\frac{1}{2}\left(\left\|\delta_{u x}^{2}-\delta_{u x}^{1}\right\|^{2}+\left\|\delta_{u x}^{2}\right\|^{2}-\left\|\delta_{u x}^{1}\right\|^{2}\right)+\frac{1}{\epsilon}\left[U^{2}(1)-g\right]_{+} \gamma^{2}(1)=-\left(U_{x}^{1}-a\left(\Theta^{1}-\theta_{A} r\right)+\zeta \delta_{u x}^{1}, \gamma_{x}^{2}\right)
\end{aligned}
$$

Now,

$$
\begin{aligned}
& -\left(U_{x}^{1}-a\left(\Theta^{1}-\theta_{A} r\right)+\zeta \delta_{u x}^{1}, \gamma_{x}^{2}\right)=-\left(U_{x}^{0}+(\Delta t+\zeta)\left(P_{E}^{h} u_{1}\right)_{x}-a\left(\Theta^{0}+\Delta t \delta_{\theta}^{1}-\theta_{A} r\right), \gamma_{x}^{2}\right) \\
& \quad=-\left(u_{0 x}+(\Delta t+\zeta) u_{1 x}-a\left(\Theta^{0}-\theta_{A} r\right), \gamma_{x}^{2}\right)+a \Delta t\left(\delta_{\theta}^{1}, \gamma_{x}^{2}\right) \leq C+\frac{1}{2}\left\|\gamma^{2}\right\|^{2}+\frac{a^{2} \Delta t}{2 \zeta}\left\|\delta_{\theta}^{1}\right\|^{2}+\frac{\zeta \Delta t}{2}\left\|\gamma_{x}^{2}\right\|^{2}
\end{aligned}
$$

where integration by parts and the hypothesis on the initial data were used. The definition of $U^{1}$, the assumption that $u_{1}(1)=0$ and the properties of $P_{E}^{h}$ yields $\gamma^{2}(1)=\left(U^{2}(1)-U^{0}(1)\right) /(\Delta t)^{2}$. Thus,

$$
\begin{aligned}
{\left[U^{2}(1)-g\right]_{+} \gamma^{2}(1)=\frac{1}{(\Delta t)^{2}}\left[U^{2}(1)-g\right]_{+}\left(U^{2}(1)-g+\right.} & \left.g-U^{0}(1)\right) \\
& =\frac{1}{(\Delta t)^{2}}\left[U^{2}(1)-g\right]_{+}^{2}-\frac{1}{(\Delta t)^{2}}\left[U^{2}(1)-g\right]_{+}\left(U^{0}(1)-g\right) \geq 0
\end{aligned}
$$

Therefore,

$$
\begin{aligned}
\frac{1}{2}\left(\left\|\delta_{\theta}^{2}-\delta_{\theta}^{1}\right\|^{2}+\left\|\delta_{\theta}^{2}\right\|^{2}\right)+\Delta t\left\|\delta_{\theta x}^{2}\right\|^{2}+k \Delta t\left(\delta_{\theta}^{2}(1)\right)^{2}+\frac{1}{2}\left\|\gamma^{2}\right\|^{2}+\frac{\zeta \Delta t}{2}\left\|\gamma_{x}^{2}\right\|^{2} & \\
& +\frac{1}{2}\left(\left\|\delta_{u x}^{2}-\delta_{u x}^{1}\right\|^{2}+\left\|\delta_{u x}^{2}\right\|^{2}\right) \leq \frac{1}{2}\left(\left\|\delta_{\theta}^{1}\right\|^{2}+\left\|\delta_{u x}^{1}\right\|^{2}\right)+C+\frac{a^{2} \Delta t}{2 \zeta}\left\|\delta_{\theta}^{1}\right\|^{2} .
\end{aligned}
$$


From Corollary 4.2 we have that $\Delta t\left\|\delta_{\theta}^{1}\right\|^{2} \leq C$ and we need to show that $\left\|\delta_{\theta}^{1}\right\|$ is bounded. Writing equation (3.1) for $n=1$ yields, $\forall w \in S_{E}^{h}$,

$$
\left(\delta_{\theta}^{1}, w\right)+\left(\Delta t \delta_{\theta x}^{1}+\Theta_{x}^{0}, w_{x}\right)+a\left(\delta_{u x}^{1}, w\right)+k \Theta^{1}(1) w(1)=0 .
$$

Since $\left(\Theta_{x}^{0}, w_{x}\right)=\left(\theta_{0 x}+c \theta_{A}, w_{x}\right)=c \theta_{A} w(1)-\left(\theta_{0 x x}, w\right)$ we find that, $\forall w \in S_{E}^{h}$,

$$
\left(\delta_{\theta}^{1}, w\right)+\Delta t\left(\delta_{\theta x}^{1}, w_{x}\right)+a\left(\delta_{u x}^{1}, w\right)+\left(k \Theta^{1}(1)+c \theta_{A}\right) w(1)-\left(\theta_{0 x x}, w\right)=0 .
$$

Taking $w=\delta_{\theta}^{1}$ and observing that $\Theta^{1}(1)=\Delta t \delta_{\theta}^{1}(1)-c \theta_{A} / k$ we obtain

$$
\left\|\delta_{\theta}^{1}\right\|^{2}+\Delta t\left\|\delta_{\theta x}^{1}\right\|^{2}+\Delta t k \delta_{\theta}^{1}(1)^{2}=\left(\theta_{0 x x}-a \delta_{u x}^{1}, \delta_{\theta}^{1}\right) \leq\left\|\theta_{0 x x}\right\|^{2}+a^{2}\left\|\delta_{u x}^{1}\right\|^{2}+\frac{1}{2}\left\|\delta_{\theta}^{1}\right\|^{2}
$$

and the statement of the theorem follows.

Remark 4.4. Note that, similarly to the continuous case, the proof of Theorem 4.1 yields

$$
\frac{\hat{E}^{n}-\hat{E}^{n-1}}{\Delta t} \leq 0 \quad n=2, \ldots, N
$$

where

$$
\hat{E}^{n}=\frac{1}{2}\left(\left\|\Theta^{n}\right\|^{2}+\left\|\delta_{u}^{n}\right\|^{2}+\left\|U_{x}^{n}\right\|^{2}+\frac{1}{\epsilon}\left[U^{n}(1)-g\right]_{+}^{2}\right)+a \theta_{A}\left(r, U_{x}^{n}\right) .
$$

\section{Convergence}

We proceed to prove that the numerical method converges.

Given $\xi \in C^{\infty}(0, T)$, we define the piecewise, constant in time, functions

$$
\theta^{h, \Delta t}(t)=\sum_{i=0}^{N} \Theta^{i} \chi^{i}(t), \quad u^{h, \Delta t}(t)=\sum_{i=0}^{N} U^{i} \chi^{i}(t), \quad \xi^{\Delta t}(t)=\sum_{i=1}^{N} \xi^{i-1} \chi^{i}(t),
$$

where $\chi^{i}$ is the characteristic function of $[(i-1) \Delta t, i \Delta t)$, and $\xi^{i}=\xi\left(t_{i}\right)$, and the piecewise linear, continuous in time, functions $\hat{u}^{h, \Delta t}, \hat{\theta}^{h, \Delta t}$ such that $\hat{u}^{h, \Delta t}(i \Delta t)=U^{i}, \hat{\theta}^{h, \Delta t}(i \Delta t)=\Theta^{i}$. We introduce the operators

$$
\begin{array}{r}
\delta u^{h, \Delta t}(t)=\frac{u^{h, \Delta t}(t)-u^{h, \Delta t}(t-\Delta t)}{\Delta t} \\
\gamma u^{h, \Delta t}(t)= \begin{cases}\frac{\delta u^{h, \Delta t}(t)-\delta u^{h, \Delta t}(t-\Delta t)}{\Delta t} & t \in[\Delta t, T], \\
0 & t \in[0, \Delta t) .\end{cases}
\end{array}
$$

It follows, from Theorems 4.1, and 4.3 and Corollary 4.2, that we can extract subsequences such that, as $h$, $\Delta t \rightarrow 0$,

$$
\begin{gathered}
\theta^{h, \Delta t} \rightarrow \theta, \quad \hat{\theta}^{h, \Delta t} \rightarrow \theta \text { in } L^{\infty}\left(0, T ; H_{E}^{1}(I)\right) \text { weakly*, } \\
u^{h, \Delta t} \rightarrow u, \quad \hat{u}^{h, \Delta t} \rightarrow u, \quad \delta u^{h, \Delta t} \rightarrow u_{t} \text { in } L^{\infty}\left(0, T ; H_{E}^{1}(I)\right) \text { weakly*, } \\
\delta \theta^{h, \Delta t} \rightarrow \theta_{t}, \quad \gamma u^{h, \Delta t} \rightarrow u_{t t} \text { in } L^{\infty}\left(0, T ; L^{2}(I)\right) \text { weakly*, } \\
\delta \theta^{h, \Delta t} \rightarrow \theta_{t}, \quad \gamma u^{h, \Delta t} \rightarrow u_{t t} \text { in } L^{2}\left(0, T ; H_{E}^{1}(I)\right) \text { weakly }, \\
\theta^{h, \Delta t}(1, \cdot) \rightarrow \theta(1, \cdot) \text { in } L^{\infty}(0, T) \text { weakly*. }
\end{gathered}
$$


Furthermore, $\hat{u}^{h, \Delta t}(1, \cdot) \rightarrow u(1, \cdot), \xi^{\Delta t} \rightarrow \xi$ in $L^{2}(0, T)$ strongly. Since

$$
\left\|\hat{u}^{h, \Delta t}(1, \cdot)-u^{h, \Delta t}(1, \cdot)\right\|_{L^{2}(0, T)}^{2} \leq \Delta t \sum_{i=1}^{N}\left(U^{i}(1)-U^{i-1}(1)\right)^{2} \leq \Delta t \sum_{i=1}^{N}\left\|U_{x}^{i}-U_{x}^{i-1}\right\|^{2}
$$

and

$$
\begin{aligned}
\left\|\left[u^{h, \Delta t}(1, \cdot)-g\right]_{+}-[u(1, \cdot)-g]_{+}\right\| \leq\left\|u^{h, \Delta t}(1, \cdot)-u(1, \cdot)\right\| & \\
& \leq \|\left[u^{h, \Delta t}(1, \cdot)-\hat{u}^{h, \Delta t}(1, \cdot)\left\|_{L^{2}(0, T)}+\right\| \hat{u}^{h, \Delta t}(1, \cdot)-u(1, \cdot) \|_{L^{2}(0, T)}\right.
\end{aligned}
$$

the stability estimate given in Theorem 4.1 implies that

$$
\left[u^{h, \Delta t}(1, \cdot)-g\right]_{+} \rightarrow[u(1, \cdot)-g]_{+} \text {in } L^{2}(0, T) \text { strongly. }
$$

Let $\eta \in H_{E}^{1}(I)$ be arbitrary. We set $w=P_{E}^{h} \eta$ and multiply (3.1) by $\Delta t \xi^{n-1}$ and sum over $n, n=1, \ldots, N$. Then, recalling the strong convergence of $w$,

$$
\int_{0}^{T}\left(\left(\delta \theta^{h, \Delta t}, w\right)+\left(\theta_{x}^{h, \Delta t}, w_{x}\right)+a\left(\delta u_{x}^{h, \Delta t}, w\right)\right) \xi^{\Delta t}(t) \mathrm{d} t+w(1) \int_{0}^{T} k \theta^{h, \Delta t}(1, t) \xi^{\Delta t}(t) \mathrm{d} t=0
$$

Passing to the limit as $h, \Delta t \rightarrow 0$ we find that

$$
\int_{0}^{T}\left(\left(\theta_{t}, \eta\right)+\left(\theta_{x}, \eta_{x}\right)+a\left(u_{x t}, \eta\right)+k \theta(1, t) \eta(1)\right) \xi(t) \mathrm{d} t=0
$$

which implies

$$
\left(\theta_{t}, \eta\right)+\left(\theta_{x}, \eta_{x}\right)+a\left(u_{x t}, \eta\right)+k \theta(1, t) \eta(1)=0
$$

a.e. in $(0, T)$. Next, we take $v=P_{E}^{h} \chi, \chi \in H_{E}^{1}(I)$, and multiply equation (3.2) by $\Delta t \xi^{n-1}$ and sum over $n$, $n=2, \ldots, N$, to obtain

$\int_{\Delta t}^{T}\left(\left(\gamma u^{h, \Delta t}, v\right)+\left(u_{x}^{h, \Delta t}+\zeta \delta u_{x}^{h, \Delta t}-a \theta^{h, \Delta t}+a \theta_{A} r, v_{x}\right)\right) \xi^{\Delta t}(t) \mathrm{d} t+\frac{1}{\epsilon} \int_{\Delta t}^{T}\left[u^{h, \Delta t}(1, t)-g\right]_{+} v(1) \xi^{\Delta t}(t) \mathrm{d} t=0$.

Passing to the limit as $h, \Delta t \rightarrow 0$ it results

$$
\int_{0}^{T}\left(\left(u_{t t}, \chi\right)+\left(u_{x}+\zeta u_{x t}-a \theta+a \theta_{A} r, \chi_{x}\right)\right) \xi(t) \mathrm{d} t+\frac{1}{\epsilon} \int_{0}^{T}[u(1, t)-g]_{+} \chi(1) \xi(t) \mathrm{d} t=0
$$

and therefore,

$$
\left(u_{t t}, \chi\right)+\left(u_{x}+\zeta u_{x t}-a \theta+a \theta_{A} r, \chi_{x}\right)+\frac{1}{\epsilon}[u(1, t)-g]_{+} \chi(1)=0
$$

a.e. in $(0, T)$. Because $\delta \theta^{h, \Delta t} \rightarrow \theta_{t}$ in $L^{2}\left(0, T ; H_{E}^{1}(I)\right)$ weakly implies that $\Theta^{0}(\cdot) \rightarrow \theta(\cdot, 0)$ in $H_{E}^{1}(I)$ weakly and we have that $\Theta^{0} \rightarrow \theta_{0}+\theta_{A} r$ in $H_{E}^{1}(I)$ strongly, we conclude that $\theta(x, 0)=\theta_{0}(x)+\theta_{A} r$. In the same way, the convergence of $\delta u^{h, \Delta t}$ and $\gamma u^{h, \Delta t}$ leads to $u(x, 0)=u_{0}(x)$ and $u_{t}(x, 0)=u_{1}(x)$.

\section{Numerical SIMUlations}

We now present some numerical calculations.

In our computations, we let $a=0.017, g=0.1, \theta_{A}=10, h=1 / 250$ and $\Delta t=0.001$. The initial data were $\theta_{0}(x)=\theta_{A}(\cos 2 \pi x-\sin 0.5 \pi x), u_{0}(x)=0$ and $u_{1}(x)=20 x(x-1)^{2}$. Numerical integration was used to compute 

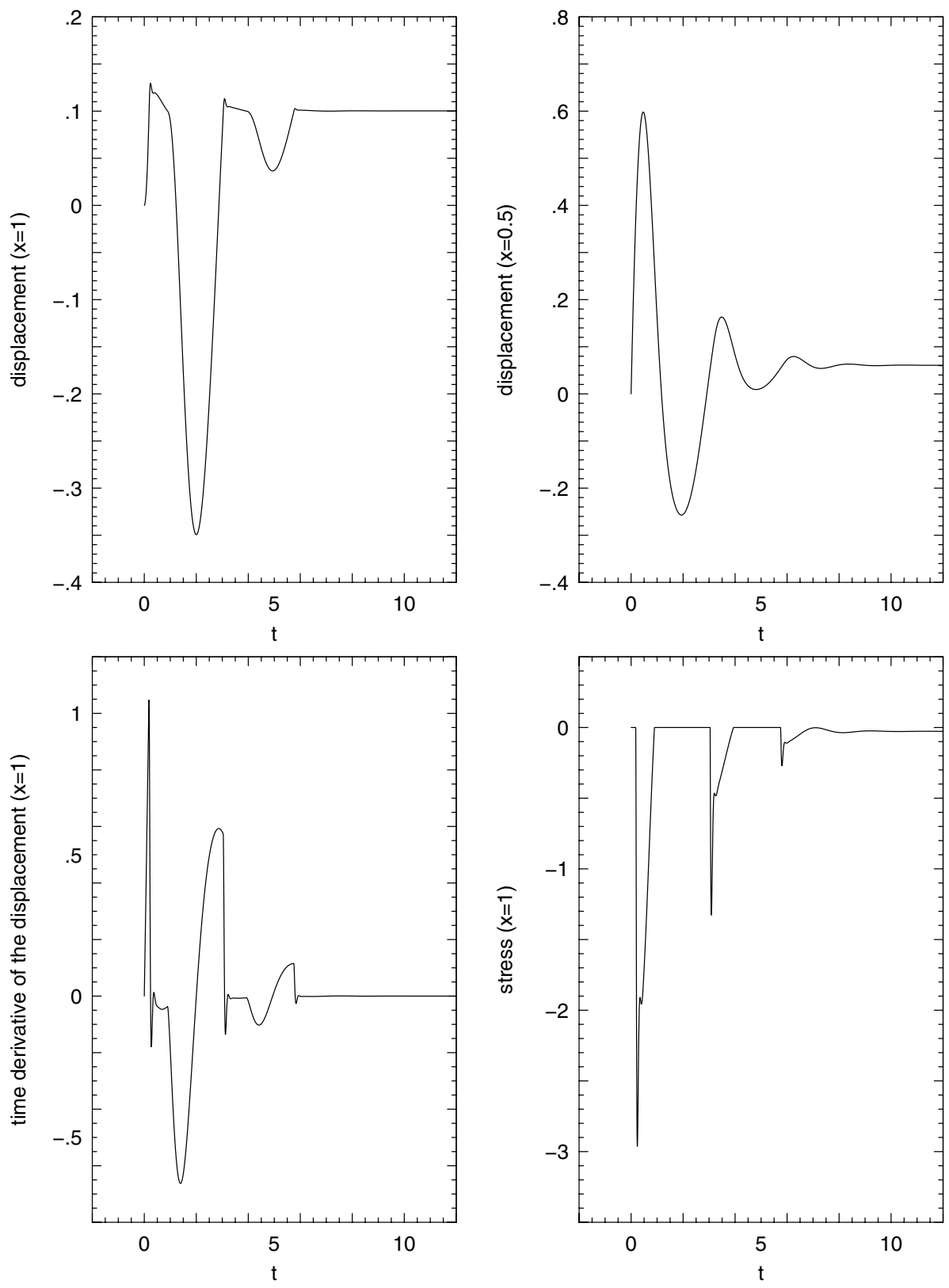

Figure 1. $\epsilon=0.01, k=1, \zeta=0.2$.

the matrix $M$. For each experiment we show the displacement at $x=1$ and $x=0.5$ and the velocity of the deformation and the stress at the contact point.

First, we took $\epsilon=0.01, k=1$ and $\zeta=0.2$ and then we increased the coefficient of viscosity to $\zeta=1$. When $\zeta=0.2$, initially, the solution is oscillatory with intervals of contact with penetration $(u(1)>g)$ and separation. In both experiments, we observe contact at later times. The rough behaviour of $u_{t}$ is due to the fact that the obstacle is almost rigid. See Figures 1 and 2. 

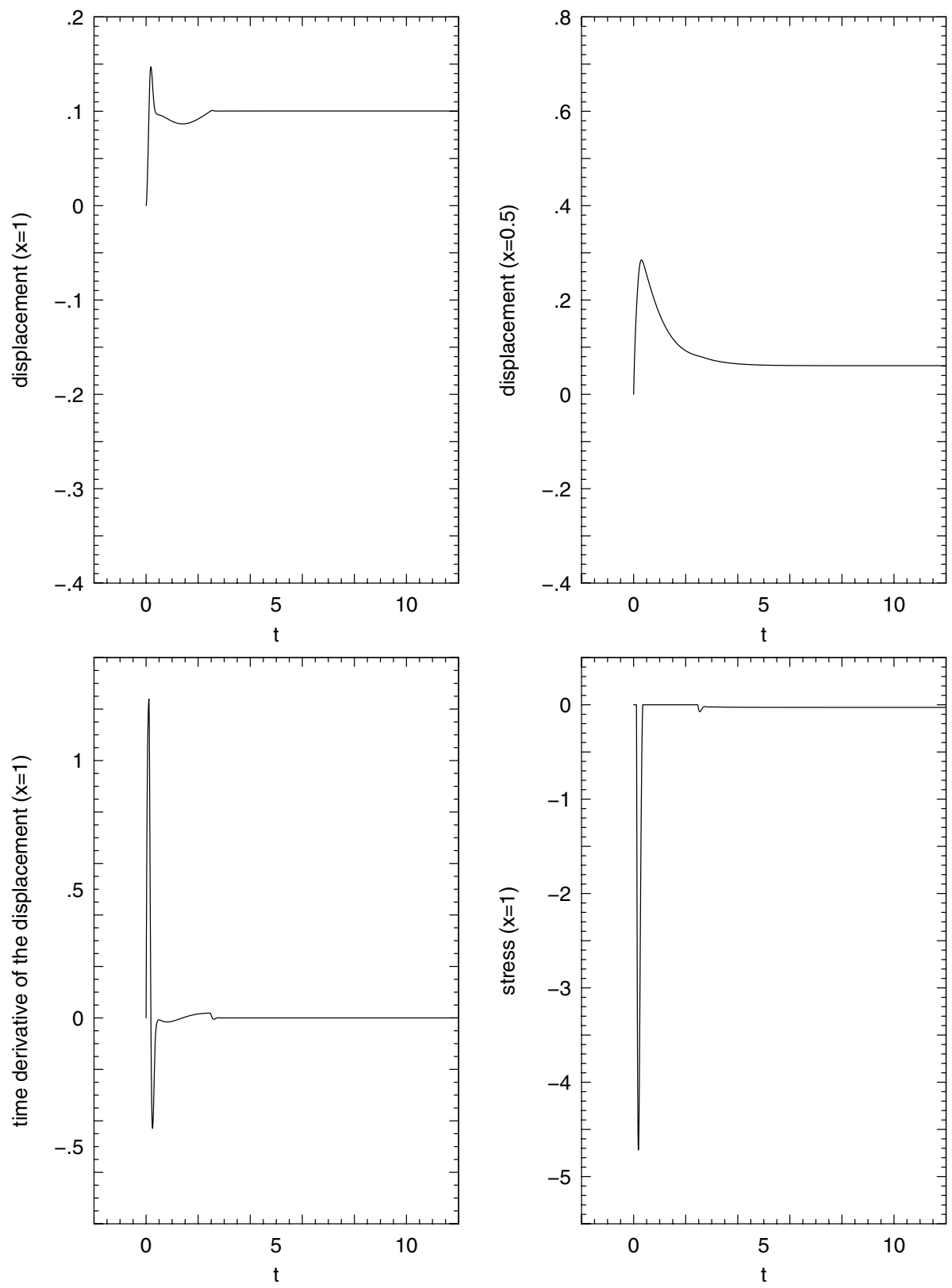

Figure 2. $\epsilon=0.01, k=1, \zeta=1$.

In the third and fourth experiments, we let $\epsilon=0.01, k=100$, with coefficients of viscosity $\zeta=0.2$ and $\zeta=1$, respectively. Again, during some time, we saw intervals of penetration and separation but the system evolved towards a state with no contact with the obstacle with the stress at the contact point equal to zero (Fig. 3 ). 

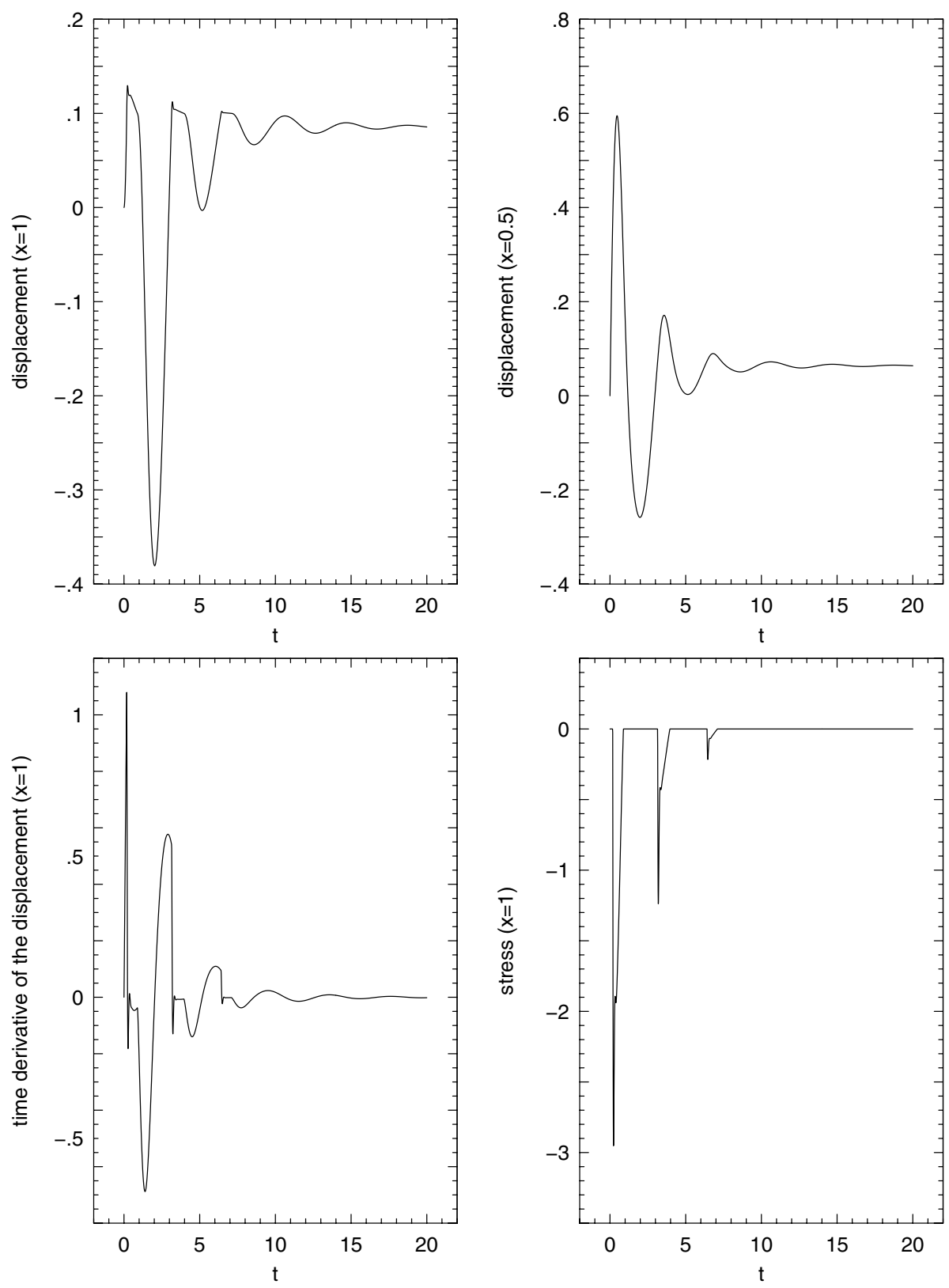

Figure 3. $\epsilon=0.01, k=100, \zeta=0.2$.

Figure 4 shows the results for the last experiment, when the obstacle is more elastic with $\epsilon=1, k=1$ and $\zeta=0.2$. As expected, the solution is oscillatory and smoother. At first, it is seen a large penetration in the obstacle with the oscillations damping out as time progresses. 

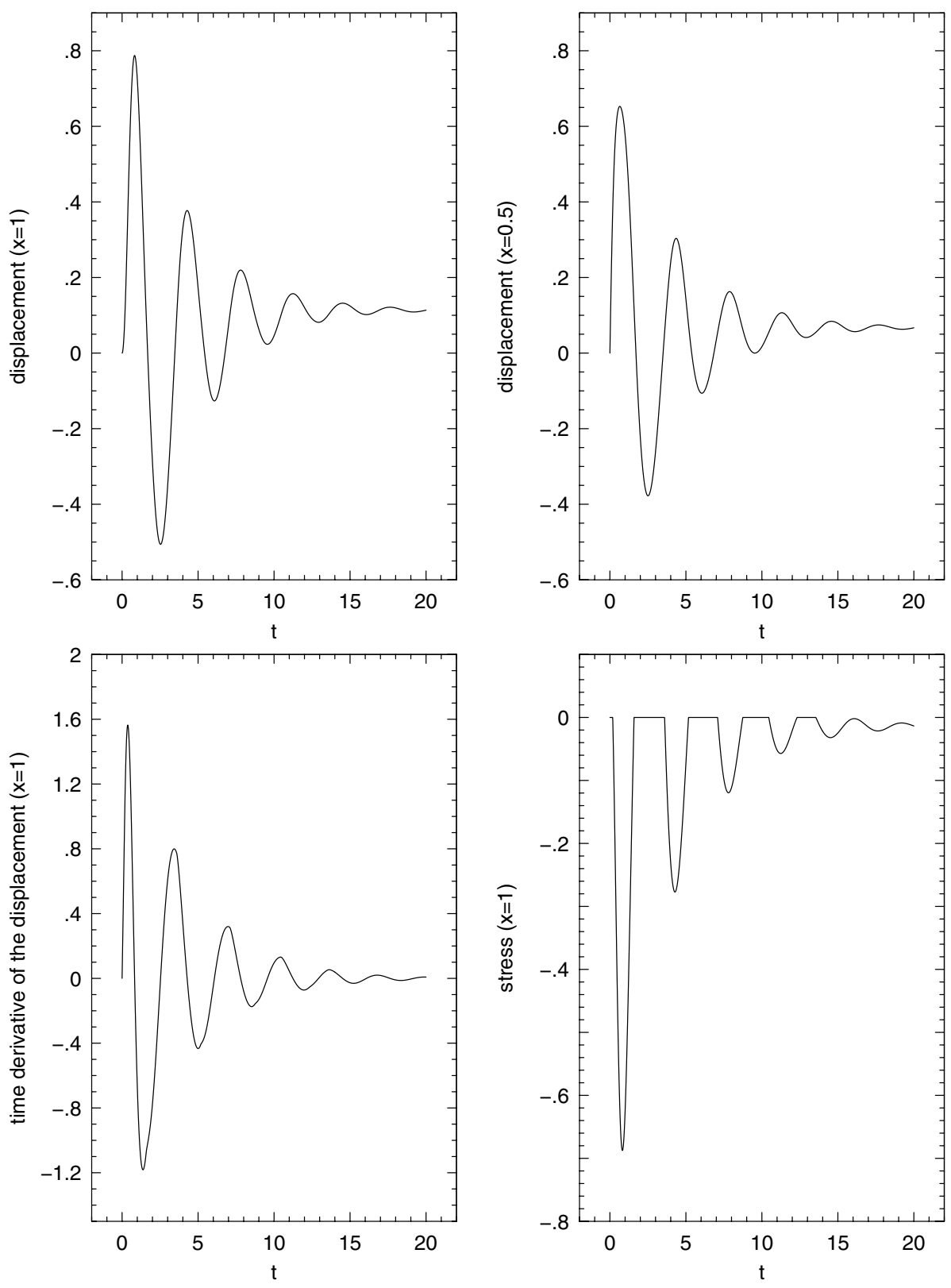

Figure 4. $\epsilon=1, k=1, \zeta=0.2$.

In all experiments, convergence towards a stationary solution, as described in [2], seems to take place. When $\zeta=1$, a rapid stabilization is observed. Also, as reported previously by Copetti and French [3] and Copetti [2] to the quasi-static problem, the temperature profiles were virtually identical when $\zeta$ and $\epsilon$ changed. The decay of the energy when $\epsilon=0.01$ and $k=1$ is shown in Figure 5 where $\ln \left(\hat{E}^{n}+0.01\right)$ is plotted against time.

Acknowledgements. The author is thankful to the referees for valuable suggestions that improved the manuscript. 

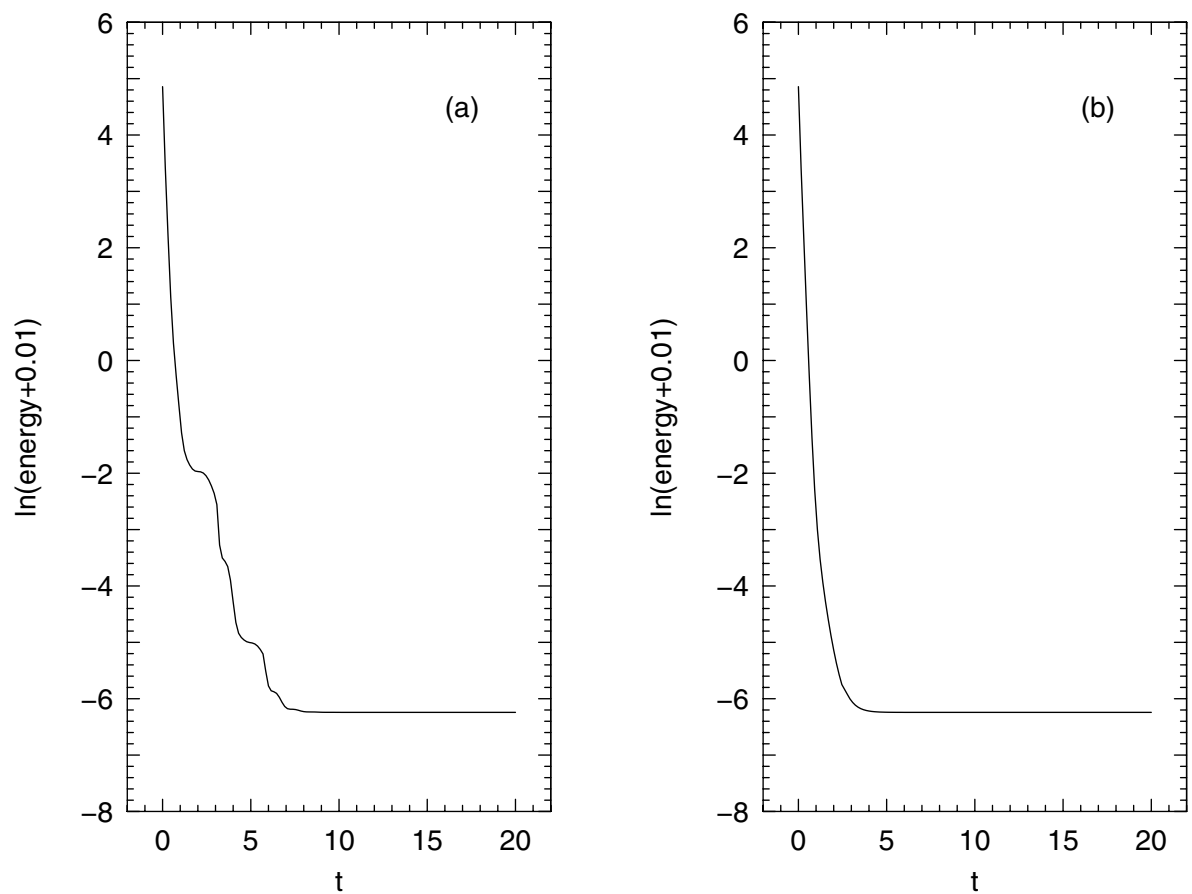

Figure 5. The time evolution of the energy when $\epsilon=0.01, k=1, \zeta=0.2$ (a) and $\zeta=1$ (b).

\section{REFERENCES}

[1] D.E. Carlson, Linear thermoelasticity, in Handbuch der physik, C. Truesdell Ed., VIa/2 (1972) 297-345.

[2] M.I.M. Copetti, A one-dimensional thermoelastic problem with unilateral constraint. Math. Comp. Simul. 59 (2002) $361-376$.

[3] M.I.M. Copetti and D.A. French, Numerical solution of a thermoviscoelastic contact problem by a penalty method. SIAM J. Numer. Anal. 41 (2003) 1487-1504.

[4] W.A. Day, Heat conduction with linear thermoelasticity. Springer, New York (1985).

[5] C. Eck, Existence of solutions to a thermo-viscoelastic contact problem with Coulomb friction. Math. Mod. Meth. Appl. Sci. 12 (2002) 1491-1511.

[6] C. Eck and J. Jarušek, The solvability of a coupled thermoviscoelastic contact problem with small Coulomb friction and linearized growth of frictional heat. Math. Meth. Appl. Sci. 22 (1999) 1221-1234.

[7] C.M. Elliott and T. Qi, A dynamic contact problem in thermoelasticity. Nonlinear Anal. 23 (1994) 883-898.

[8] S. Jiang and R. Racke, Evolution equations in thermoelasticity. Chapman \& Hall/ CRC (2000).

[9] J.U. Kim, A one-dimensional dynamic contact problem in linear viscoelasticity. Math. Meth. Appl. Sci. 13 (1990) 55-79.

[10] K.L. Kuttler and M. Shillor, A dynamic contact problem in one-dimensional thermoviscoelasticity. Nonlinear World 2 (1995) $355-385$.

[11] M. Schatzman and M. Bercovier, Numerical approximation of a wave equation with unilateral constraints. Math. Comp. $\mathbf{5 3}$ (1989) $55-79$. 